\title{
The Monitoring System Research of the River Water Quality Based on GIS
}

\author{
Liwei Liu* \\ College of Biology and the Environment, Nanjing Forestry University, Nanjing, JiangSu, China
}

\begin{abstract}
In this paper, the GIS technology and river water quality monitoring were integrated to design an intelligent water environment monitoring and disaster warning system. The designed system can not only provide web publishing service for water environment monitoring, but it can also prompt to the user the possibility of water disaster according to the change of some important parameters in water, to realize the warning in advance. That can make the monitoring system more intelligent to avoid water disaster.
\end{abstract}

Keywords: GIS, monitoring system, the river, warning, water quality.

\section{INTRODUCTION}

Geographic information system (GIS) is based on the computer-aided collection of the geographical spatial location information including the collection, storage, management, analysis, visualization and application of software tools. Particularly, it is much suitable for digital storage of spatial elements building, analysing and querying that are based on the spatial position relationships. Infrastructure management information system was established based on GIS, which can easily form various targets of the infrastructure digital storage, and can perform a detailed analysis of the relationship between the targets and can also assist in the operations maintenance planning etc. [1].

GIS and the Internet of Things have changed the traditional way of information collection and information processing, that is the processing of information from the numerical fields into the space. According to the actual need, GIS technology of the geographical location and the related properties can organically deal with the science accurately, and illustrate the output results to the user. Then, the user, through its unique spatial analysis function and visual expression, will be a combination of all kinds of decisions that can be performed [2]. At present, the Internet and GIS used in environmental protection and governance, environmental monitoring, environment disaster monitoring and controlling, besides being used in many other fields such as ecological protection, utilization of resources, obtained the preliminary application and so on.

So, using the latest development of GIS for real-time monitoring of water environment and disaster warning based on the monitoring of water environment problems and the insufficiency, is of great significance.

\footnotetext{
*Address correspondence to these authors at the College of Biology and the Environment, Nanjing Forestry University, Nanjing, JiangSu, China; Tel: +86-139-1478-1987; E-mails: 76560136@qq.com
}

\section{MATERIAL AND METHODS}

In the past, the environmental protection department is mainly carried through the simulation analysis that was performed based on the acquisition of data to realize the river water quality monitoring. The test sites were more dispersed, and water quality data, only one-sided that could not reflect the whole condition of river water quality. In addition, transferring technical analysis was far behind than the requested. That led to water quality monitoring results always behind the real situation. At that time monitoring data could not reflect the dynamic condition of the river water quality in a timely manner [3]. So, designing a river water quality system that can reflect real-time water quality is very essential. This article will put forward a kind of river water quality dynamic monitoring system based on embedded technology, communication technology and GIS technology.

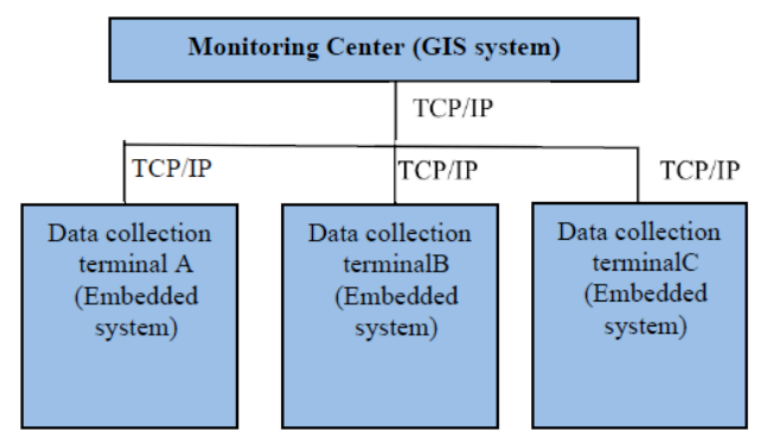

Fig. (1). Total system framework map. TCP means transmission control protocol, ip means internet protocol.

The whole system consists of two parts: monitor center and data acquisition terminal. Monitoring center is the server of the whole system. Data acquisition terminal is an embedded system that carries on field data acquisition for the river water quality assessment after GIS system running. The river water quality is monitored on a coverage range between GIS system and data acquisition terminal via TCP/IP for Internet 
communications [4]. Data acquisition terminal is via TCP/IP to realize data for a reliable transmission over a long distance; the GIS monitoring center receives all end data including quality data of river water, storage data of the water quality, analysis data, and management data, query data, display data, and management data of all collection terminals.

\section{The Realization of GIS Monitoring System}

GIS based geography, geometry and computer science, are using graphics and database technology, in which the spatial information and spatial information acquisition, storage, analysis, management and display system are included.

In this design, GIS for river water quality was implemented, according to the data storage, analysis, simulation, and implementation of river water quality monitoring [5]. The whole system consists of database, GIS visualization interface and water quality model. The system block diagram is shown in Fig. (2).

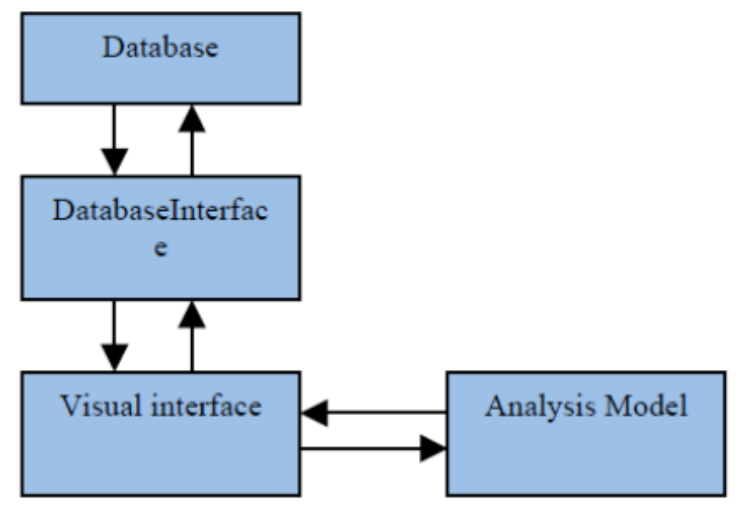

Fig. (2). GIS system model.

GIS visual interface directly displays space object and space properties such as location, spatial distribution; and additional GIS visual also displays space object attribute data through the association space. The attribute data of spatial and non-spatial is managed through spatial and non-spatial database. Spatial attribute database of GIS spatial data management includes topographic map, various thematic maps, basin, embedded system terminal, pollution source and shape, etc. Non-spatial attribute database includes service related database, for the management of a variety of spatial attribute data, includes the water quality monitoring data (such as river flow, flow rate, dissolved oxygen DO, etc.), and statistics and social attribute data (such as economic conditions, industrial layout and the water quality standard, etc.), etc. [5]. GIS visual interface provides various standard databases that read the spatial data and non-spatial data. At the same time, GIS realizes the query, positioning, analysis, simulation, and early warning, and other functions through the interaction with the database.

Water quality model pollutants change rule in water environment and influence factors of the mutual relationship between mathematical descriptions; it is one of the important means of water quality monitoring. In recent years, a variety of research and application of the multivariable comprehensive water quality models have been developed, such as the QUAL model series of the national environmental protection development bureau, series of Danish institute developed MIKE hydrodynamic model etc. [6]. The complex structure of water quality model is described in great detail with respect to many parameters such as, pollutants migration and transformation process in the water. According to the actual need for a comprehensive water quality design, this model is a simplified, zero dimension model. And it is also a water environmental capacity model.

Water quality model of object is actually basin, which is a space object. Therefore, fully integrated design of the water quality model in GIS, becomes a part of the GIS function. GIS can directly use water quality model to simulate the water quality data, and the simulation results can be directly displayed on the GIS visualization interface. That provides expression of the deficiency of the water quality model and the GIS based on the analysis of simulated shortfalls [6].

\section{The Realization of the Embedded System}

Computer system embedded with the software and hardware is used as the control center, ensuring high integration and low cost, supporting a variety of real-time operating systems and advantages of network function.

This design adopts the embedded system for field water quality data collection, and through TCP/IP the collected data will be transmitted to the monitoring center. According to different functions, the system is divided into the processor module, storage module, data acquisition module, network module and other peripheral interfaces. The system module partition is shown in Fig. (3). Each module is composed of hardware and software, which act together to accomplish a specific function [7].

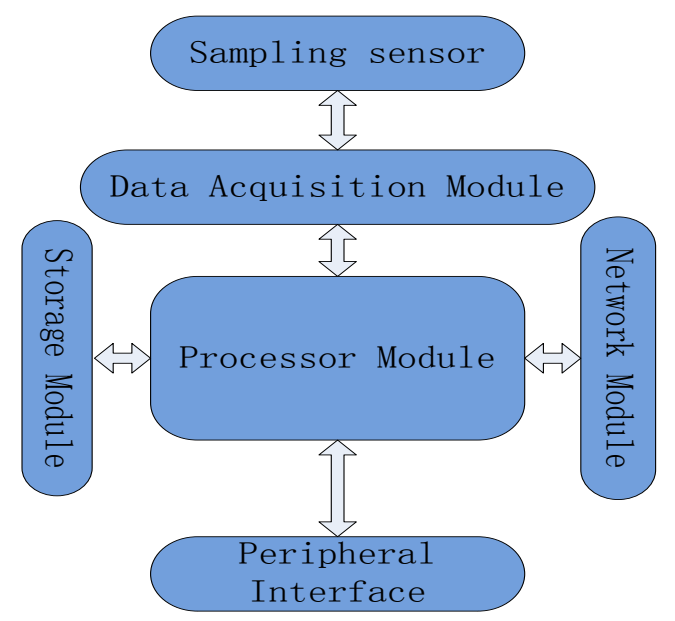

Fig. (3). Embedded structure.

Processor module is the core of the whole system; the low price, low power consumption of 32 bit ARM7TDMI and real-time operating system C/OS - II, is mainly responsible for the management of external hardware equipment, external interruption control, task scheduling and communication and information exchange between each function module. All the other software modules in the operating system have different priorities on the basis of the implementation and operation, at any time of the sleep state, the ready state, running state, waiting for state, and one of the states of 
the interrupt state. The operating system by sending is a mail message structure to control the state of the various modules.

Data storage module consisting of memory and files system, is responsible for storing programs and data. Memory uses 2M FlashRom, 6M NandFlash and 8M Sdram, among them 2M FlashRom is used to store system bootstrap, 16M NandFlash is responsible for storing programs and data, and $8 \mathrm{M}$ Sdram is responsible for the application and data storage tasks. To avoid the difference effect between data reading and writing system, file system and data storage are managed separately. At the same time, it can also provide the standard API interface as well as the introduction of high-speed read and write buffer, so as to avoid direct read and write task of NandFlash. The design has the advantage of solving the memory problem of data reading speed between CPU and Flash storage [4].

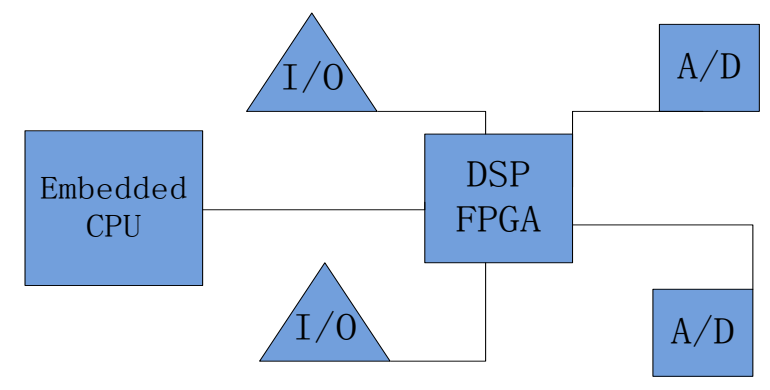

Fig. (4). Data processing logic diagram.

Sensor data acquisition module, data acquisition task and data processing tasks are responsible for a variety of data collection and processing tasks. For water quality monitoring, data from the sensor mainly includes: water quality comprehensive indexes (such as dissolved oxygen DO), the water quality pollution indicators (such as biochemical oxygen demand BOD, chemical oxygen demand (COD) and hydrological parameters (velocity and flow rate). Data acquisition task is usually in a wait state, waiting for news of control parameters. Mainly, the system adopts frequency control parameters, the choice of channel, and start the A/D converter, etc. At the same time, the data acquisition tasks design a $4 \mathrm{~KB}$ capacity of ring type stack, for temporarily storing the sampling data [5]. Data processing I/O (I/O means data input/data output) are idle most of the time, with the same size of the stack data acquisition task, when need to immediately send data to invoke the CPU (Fig. 4).

Network module used 8019 as card chip, embedded TCP/IP protocol and network tasks, mainly completed the data sending through GIS system for communication and monitoring canter. This system with reference to the UNIX TCP/IP protocol, implements all the basic TCP/IP protocols. The TCP/IP is divided into application layer, transport layer, network layer and data link layer; the network layer is composed of TP protocol and ICMP (Internet Control Message Protocol) protocol, and data link layer is composed of network card driver and the ARP (Address Resolution Protocol) protocol. Operations are isolated from each other between each layer, by calling the API (Application Programming Interface) interface function for communication, based on the need to deal with data transmitted to the upper or lower level agreement.

\section{An Embedded System Running Process}

The embedded system will be started with the FlashRom /the BootLoader to initialize the CPU and network card hardware from NandFlash, then picks up a copy of the operating system kernel and application tasks on Sdram.

After the operating system gains control of CPU, the tasks of operating system and application initialization will work immediately. Firstly, all data structure initialize, with the purpose to allocate stack space, build the message queue, set up tasks, etc. Secondly, read the system running state parameters stored in the file system; these parameters include the operation of the individual tasks, the data acquisition of sampling frequency and the IP address of the remote host. Finally, local default gateway and system login password system information, will adjust the parameter of the task.

\section{THE REALIZATION OF DYNAMIC MONITORING WATER QUALITY}

Dynamic monitoring of the implementation of the water quality is achieved through the TCP/IP field collection data of water quality in real-time transmission to the monitoring center. In order to achieve the effect of dynamic monitoring, monitoring center will receive water quality data and will display on the GIS after some analysis. The dynamic monitoring of water quality is divided into two parts: water quality data of the dynamic display of the real-time transmission and water quality data.

\section{The Rivers Pollutant Diffusion Model}

River pollution diffusion model is implemented for monitoring river water flow and pollutant concentration changes. Concentration distribution and the flow of the river, the section average flow velocity and pollutant decay rate constant and other factors are also monitored. Through the model simulation analysis, timely judging the pollutants with concentration distribution, and rivers' higher levels of warning; reduced economic loss can be achieved [6]. Detailed system model is shown in Fig. (5).

Assuming that, in some cases, the river water movement time scale is very large; at this time scale, the average pollutant concentration is in a stable condition. In this case, taking time average will handle problems according to the steadystate. And it will simplify the complexity of the model. The average steady-state model can be used to describe the flow conditions. River pollution in water body can be due to the integration of water medium with the same fluid mechanics properties. So, the contaminant particles can be calculated with water.

Assume that only a concentration gradient exists in the $\mathrm{X}$ direction. The concentration of the corresponding model is [1]:

$$
\begin{aligned}
& c=c_{0} \exp \left(-\frac{K_{x}}{u_{x}}\right) \\
& \text { then } c_{0}=\frac{Q c_{1}+Q c_{2}}{Q+q},
\end{aligned}
$$

Q: the flow of the river; 

rivers;

$\mathrm{C} 1$ : the background concentration of the pollutants in

q: sewage flow into the river;

$\mathrm{C} 2$ : certain concentration of pollutants in wastewater;

$\mathrm{C}$ : the concentration of pollutants;

Dx: longitudinal dispersion coefficient;

Ux: the section average flow velocity;

$\mathrm{K}$ : pollutants attenuation rate constant.

According to the river node, by adding symbol object in the interim layer coordinate, and then removing the unnecessary symbol object, the model formula can be calculated, and with each symbol object's location of pollutants concentration, a thematic map is created according to the pollutant concentration range, in order to quickly and easily realize the visual simulation of river pollution model.

\section{Water Quality of Real-Time Data Acquisition}

Real-time water quality data collection and transmission is achieved by a data acquisition task, data processing task, network operating system task, and file systems task.

Normally, in the waiting delay for data acquisition task, the delay time of data acquisition task is activated, and after reaching awaited state once again, the data is stored in its own stack. In this design the delay time is $10 \mathrm{~s}$, which $10 \mathrm{~s}$ is acquisitions that can change the sampling frequency of the whole system through changing the delay time of acquisition task [6].

A data acquisition is completed; there are two types of treatment: data from a stack immediate transfer mode and normal handling.

Immediate transfer mode mainly monitors the impact of the quality of river water pollution accident. When severe pollution accident occurs, it needs to quickly understand the water quality condition in time, and then the monitor center sends a sending order immediately through the network to the embedded acquisition terminal. The operating system will deal with the command judgment after sending messages live data processing tasks [7]. Data processing tasks will perform the task stack data reading in its own stack, which after reading acquisition task gets empty and goes to sleep. Then, the operating system task sends a message to activate the network task, which then will read the card data in data processing task stack buffer, after reading the TCP/IP start sending data, the stack data processing task gets empty [8]. Using this path for data acquisition in time, takes up too much system resources.

Based on normal processing method, data transmission according to the designed system is a good way. After the sampling is completed, it continues to the next task stack sampling, until the stack is full. After the expiration of the file system, the data is stored in the form of file in the Flash of the stack. Every $2 \mathrm{~h}$ network task is activated followed by read data from the Flash card buffer, which leads to sending data [9]. This way we can avoid an excessive amount of data reading and writing in data transferring and taking up system resources. Data acquisition transmission process flow chart is shown in Fig. (6).

To realize the scheduling of water environment monitoring system, routing node is responsible for data forwarding. As a gateway, the coordinator node is responsible for data uploading to the server and forwarding control command. Overall network architecture diagram is shown in the Fig. (7) below.

The network topology of this system is a tree; there are three kinds of equipments, namely the coordinator, routers, and terminals. Among the equipments, as the terminal equipment, data acquisition node is responsible for the collection of all nodes data, such as temperature and humidity data [10]; control node is responsible for terminal equipment, to close and cut each branch at implementation code that is shown in appendix at the end this paper.

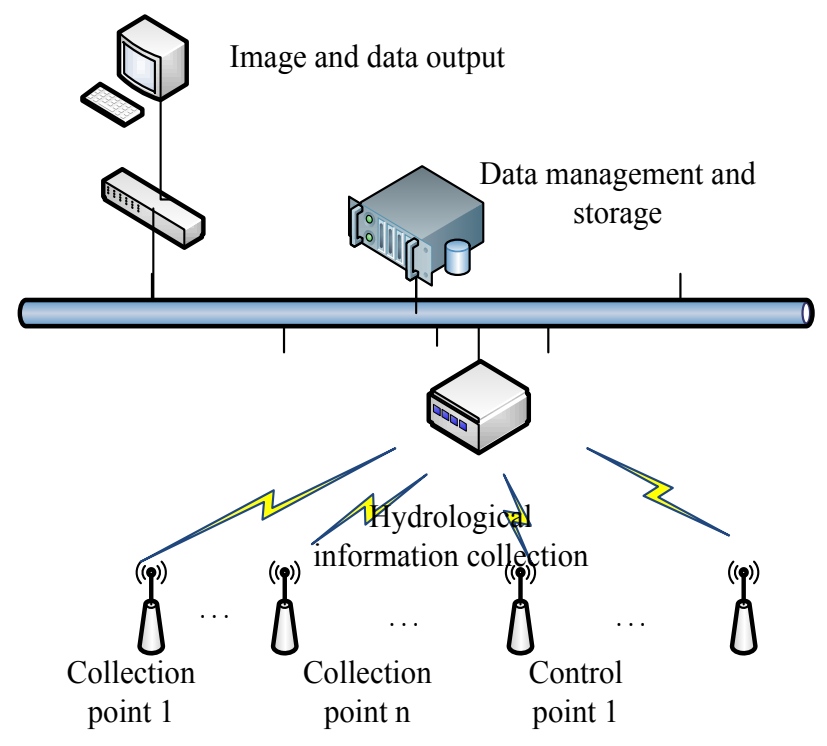

Fig. (6). Sample collection framework. 


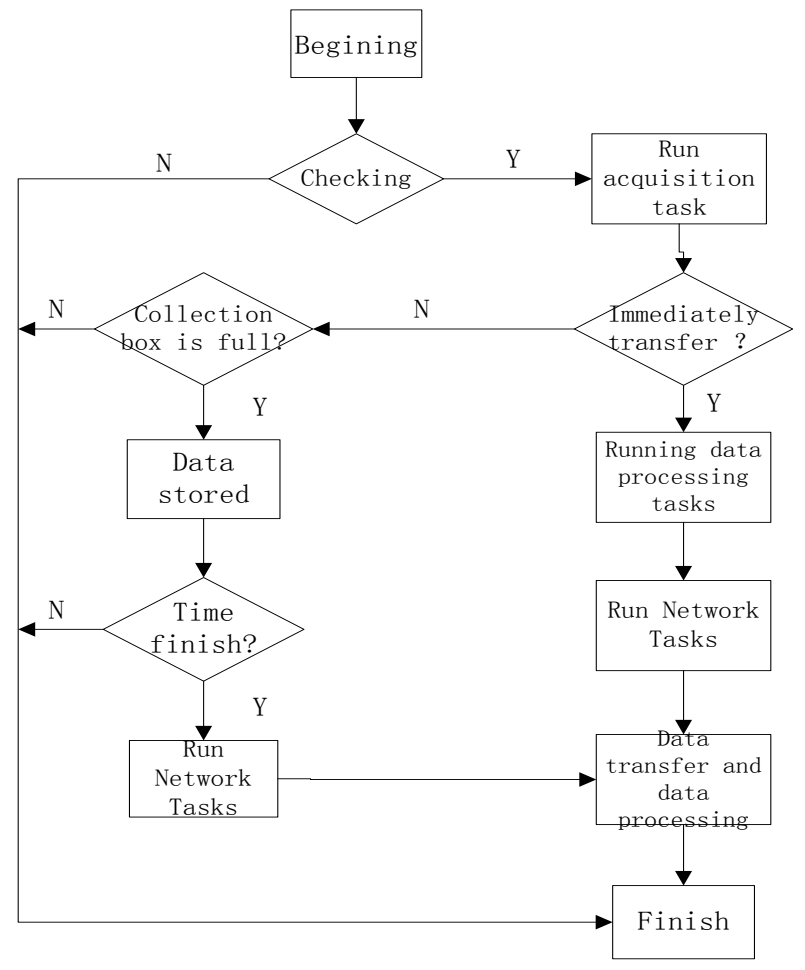

Fig. (7). Sample collection and logic diagram.

\section{The Dynamic Display of the Water Quality Data}

The dynamic display of the water quality data means water quality appears on GIS visual interface automatically after analysing and processing the test data.

After water quality monitoring center receives the data, GIS will validate the data according to certain rules, if the data matches the validation rules it will be stored in the database. Visual interface will readout the stored data through database API interfaces, and start the collecting and processing tasks. After that, the system will draw on water quality analysis models to start simulation and analysis, the simulation results and analysis data will display on the GIS visualization interface with different colours.

\section{CONCLUSION}

The monitoring system designed and proposed by us in the paper showed the following advantages: Small impact on the ecological environment of the river. Water environment monitoring data are transmitted to the base station and the control room by low power wireless channel and low radiation detecting communication protocol in sampling point without laying cable and establishing monitoring station construction.

More intensive sensor deployment. Dense deployment of multiple sensor nodes can monitor wider range of geographical distribution of each small water parameters of water environment, and improve the accuracy of parameter detection based on a large amount of redundant information of intelligent information processing.

Automated continuous monitoring, and real-time early warning. Sensor automatic intelligence can gather and pro- cess a large number of real-time data, and monitor system statistics and analyse environmental information. Finally, it draws out the pollutants concentration distribution, based on the result the spatial distribution and overweight can be drawn in a corresponding figure and visual display, and analysis of the basin water environment quality condition will be shown in a map, tracking pollutant sources.

Fast accurate positioning. GIS monitoring system intuitively show information about pollution occurrance.

Information storage and fast information querying. The collected data of various monitoring sites in the database is very convenient for data querying in time and settings adjustment.

\section{CONFLICT OF INTEREST}

The author confirms that this article content has no conflict of interest.

\section{ACKNOWLEDGEMENTS}

Declared none.

\section{APPENDIX}

try \{

serialPort $=$

(SerialPort) portId.open("SimpleReadApp", 200000);

// Waiting for the longest time serial data

\} catch (PortInUseException e1) \{

e1.printStackTrace();

\} 


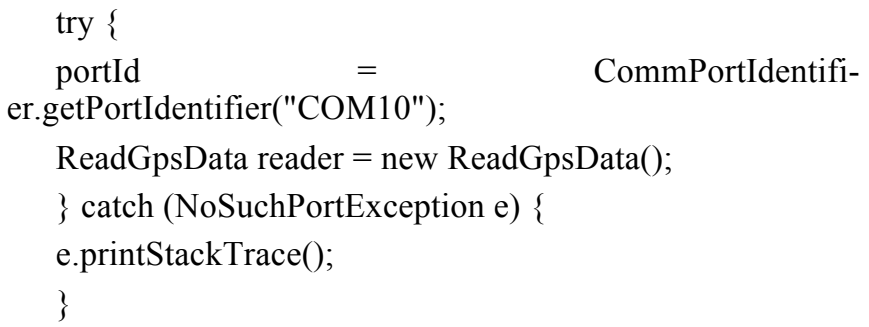

System.out.println("A serial port connection is successful!");

\} catch (UnsupportedCommOperationException e) \{

e.printStackTrace();

\}

Cutting data: if $\left((\right.$ char $)$ newData $\left.=={ }^{\prime}{ }^{\prime}\right)\{$

if (gpsData.startsWith("\#")) \{

Temp=gpsData.substring (5,7);

Hum=gpsData.substring $(11,13)$;

try \{

insertValue(Temp,Hum);

Then cut out the temperature and humidity from the data.

'Connect to the database'

Implementation of the code is as follows:

String driverName = "com.microsoft.sqlserver.jdbc.SQLServerDriver"; // Load the JDBC driver

String dbURL = "jdbc:sqlserver://localhost:1433; DatabaseName=water environment"; // Connect to the server and database test

String userName = "jstzli"; //The default user name

String userPwd = "liqiang"; //password

try \{

Class.forName(driverName);

con $=$ DriverManager.getConnection(dbURL, userName, userPwd);

System.out.println("Database connection is successful!");
// If the connection is successful, the console output Connection Successful!

\} catch (Exception e) \{

e.printStackTrace();

\}

Insert the corresponding data to the data in the table accordingly implementation code is as follows:

try\{

SimpleDateFormatdf $=$ new SimpleDateFormat("yyyy/MM/ddHH:mm:ss");

// Set the date format

System.out.println(df.format(new Date()));// new Date( ) For the current system time

String sql = "insert into

temp_hum(position,node,temp,hum,CO2,nitrogen,phosp horus, $\mathrm{PH}, \mathrm{O} 2$,in_time)

values( place)";

Statement stmt=null;// Executing SQL statements

pstmt $=$ con.prepareStatement(sql);

pstmt.setString(1, Temp);

pstmt.setString(2, Hum);

pstmt.setString(3, df.format(new Date()));

stmt $=$ con.createStatement () ;

pstmt.executeUpdate();

\}

\section{REFERENCES}

[1] X.L. Wang, "On the river ecosystem restoration," Water Power Technology, vol. 36, pp. 6-17, 2010. (In Chinese)

[2] M.G.H. Omran, and M. Mahdavi, "Objectives and principles of river ecosystem restoration," Applied Mathematics and Computation, vol. 198, pp. 19-31, 2012. (In Chinese)

[3] D.S. Wang and H.W Tan, "The impact of human activities on the river ecosystem," Science Technology and Engineering, vol. 4, pp. 298-310, 2010.

[4] J.M. Yu, and F. Zhang, "Hydraulic Stress on Ecosystems," Resources and Hydropower Engineering, vol. 34, pp. 7-12, 2009.

[5] L.B. Bronge, and B. Naslund-Landenmark, "Wetland classification for Swedish CORINE land cover adopting a semi-automatic interactive approach," Canadian Journal of Remote Sensing, vol. 28, no. 2, pp. 139-155, 2002.

[6] A. Ballard, and C. M. Brown, "Computer Vision," Englewood Cliffs. Prentice-Hall, vol. 88, pp. 99-102, 1982.

[7] P. Aplin, "Remote sensing: Land cover," Progress in Physical Geography, vol. 28, no. 2, pp. 283-293, 2004.

[8] R.J. Nicholls, M.F. Hoozenmans, and M. Marchand, "Increasing flood risk and wetland losses due to global sea level rise," Global Environmental Change, vol. 9, pp. 67-87, 1999.

[9] X.S. Wang, and X.J. Liang "Create a healthy river to maintain sustainable development," Technological Management of Land Resources, vol. 24, pp. 354-365, 2013.

[10] E.M. Patrick, "Theory and Methods for Modeling and Evaluating Multiscale Landscape Structures," International Archives of Photogrammetry and Remote Sensing, vol. 78, pp. 120-123, 2001. 